\title{
Scale Alpha and Beta of Quantitative Convergence and Chemical Reactivity Analysis in Dual Cholinesterase/Monoamine Oxidase Inhibitors for the Alzheimer Disease Treatment Using Density Functional Theory (DFT)
}

\author{
Alejandro Morales-Bayuelo, ${ }^{1,2,3}$ Rosa Baldiris, ${ }^{1}$ and Ricardo Vivas-Reyes ${ }^{1}$ \\ ${ }^{1}$ Grupo de Química Cuántica y Teórica, Universidad de Cartagena, Programa de Química, Facultad de Ciencias Exactas y Naturales, \\ 130015 Cartagena de Indias, Colombia \\ ${ }^{2}$ Departamento de Ciencias Químicas, Universidad Nacional Andres Bello, República 275, 8370146 Santiago, Chile \\ ${ }^{3}$ Corporación Universitaria Rafael Núñez, Facultad de Medicina, Cartagena, Colombia
}

Correspondence should be addressed to Alejandro Morales-Bayuelo; quicomputacional@unicartagena.edu.co and Ricardo Vivas-Reyes; rvivasr@unicartagena.edu.co

Received 13 March 2013; Accepted 27 September 2013

Academic Editors: G. Borosky, Q. Ge, M. Koyama, A. M. Lamsabhi, N. Russo, and D. Sajan

Copyright (c) 2013 Alejandro Morales-Bayuelo et al. This is an open access article distributed under the Creative Commons Attribution License, which permits unrestricted use, distribution, and reproduction in any medium, provided the original work is properly cited.

\begin{abstract}
Molecular quantum similarity descriptors and Density Functional Theory (DFT) based reactivity descriptors were studied for a series of cholinesterase/monoamine oxidase inhibitors used for the Alzheimer's disease treatment (AD). This theoretical study is expected to shed some light onto some molecular aspects that could contribute to the knowledge of the molecular mechanics behind interactions of these molecules with acetylcholinesterase (AChE) and butyrylcholinesterase (BuChE), as well as with monoamine oxidase (MAO) $A$ and $B$. The Topogeometrical Superposition Algorithm to handle flexible molecules (TGSA-Flex) alignment method was used to solve the problem of the relative orientation in the quantum similarity (QS) field. Using the molecular quantum similarity (MQS) field and reactivity descriptors supported in the DFT was possible the quantification of the steric and electrostatic effects through of the Coulomb and Overlap quantitative convergence scales (alpha and beta). In addition, an analysis of reactivity indexes is development, using global and local descriptors, identifying the binding sites and selectivity in the (cholinesterase/monoamine oxidase) inhibitors, understanding the retrodonor process, and showing new insight for drugs design in a disease of difficult control as Alzheimer.
\end{abstract}

\section{Introduction}

Alzheimer's disease $(\mathrm{AD})$ is considered a chronic disease with serious implication and many consequences. AD not only has enormous implications from the medical point of view but also has high economic costs and a negative impact on social activities within families and work places of the $\mathrm{AD}$ affected patients. $\mathrm{AD}$ is clinically characterized by progressive loss of memory and a decline in language skills and other cognitive impairments that inexorably leads to incapacitation and finally to death [1-7].
$\mathrm{AD}$ is characterized by the development of amyloid plaques and congophilic angiopathy in the brain cortex and hippocampus; it has been accepted for long as the most important pathological feature in $\mathrm{AD}$. These plaques consist of aggregated amyloid- $\beta$-peptide $(\mathrm{A} \beta)$ deposits, $\tau$-protein aggregation, oxidative stress, dyshomeostasis of biometals, and low acetylcholine (ACh) levels and plays significant role in the pathophysiology of the disease [7]. Nowadays, the most used therapeutic treatment against $\mathrm{AD}$ involves the use of acetylcholinesterase inhibitors (AChEIs) that improve AD symptoms by AChE inhibition [8-12]. 


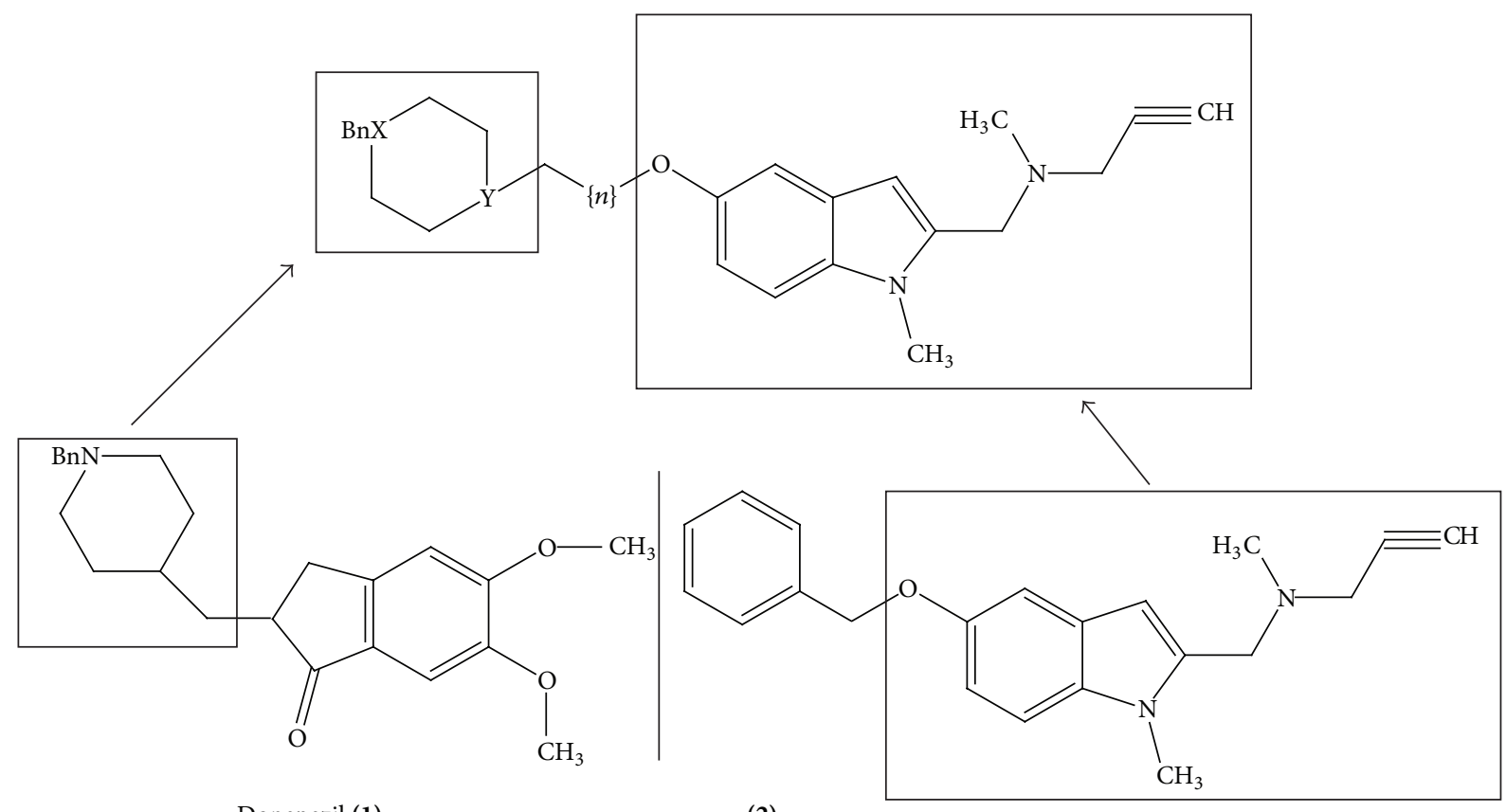

Donepezil (1)

(2)

FIGURE 1: Conjunctive approach of acetylcholinesterase inhibitor (AChEIs) donepezil (1) with the indolyl propargylamino moiety of the monoamino oxidase (MAO) $A$ and $B$ inhibitor N-[(5-benzyloxy-1-methyl-1H-indol-2-yl)methyl]-N-methylprop-2-yn-1-amine (2). Forming the IMAO/IAChe hybrids [13].

The reversible AChE inhibition has become the promising target for the treatment of $\mathrm{AD}$ which is mainly associated with low in vivo AChE levels. But due to the multifactorial nature of $\mathrm{AD}$, researchers are nowadays focusing on the improvement of new curative strategies that could help to improve and enlarge their biological profile beyond their ability to act only as AChEIs. The multitargetdirected ligand (MTDL) approach has been investigated by many researches groups [13-16]. For instance Bolea and coworkers recently have synthesized a multitarget-directed set of ligands with a variety of molecules acting on very diverse targets. Among these hybrid compounds, molecule 5 (see Table 1) is the most potent and promising multitarget inhibitor of this molecular set due to its dual inhibition either to $\mathrm{AChE}$ or $\mathrm{BuChE}$ in the same submicromolar level [13]. This new family of MTDL is able to interact with $\mathrm{AChE}$ and butyrylcholinesterase $(\mathrm{BuChE})$ and with monoamine oxidase (MAO) $A$ and $B$ (Figure 1). These compounds have been designed using a conjunctive approach that combines the benzylpiperidine moiety of the AChE inhibitor donepezil and the indolyl propargylamino moiety of the MAO inhibitor N-[(5-benzyloxy-1-methyl-1H-indol-2yl)methyl]-N-methylprop-2-yn-1-amine connected through a bond oligomethylene [13].

This molecular set is likely to act in dual way in AChE inhibitors binding site. In fact the possibility of targeting either catalytic active site (CAS) or peripheral anionic site (PAS) of AChE depends on the length of the bond. The length of the tether that connects the two main structural fragments of the novel hybrids has a significant effect on the binding to MAO, whereas it seems to have slight impact on the inhibitory activity against $\mathrm{AChE}$ and BuChE [13]. The outcome obtained by Bolea et al. shows the relevant role played by the 1-benzylpiperidin-4-yl unit on the AChE inhibitory activity, suggesting that this moiety is the main factor in mediating the binding AChE [13].

The experimental evidence in this study is supported by kinetic studies and theoretical molecular modeling. These two approaches support the dual binding site to AChE, which explains adequately the inhibitory effect exerted on $\mathrm{A} \beta$ aggregation. The results found by Bolea et al. suggest that the new compounds are multitarget drug candidates with possibly high potential impact for AD therapy [13]. Due to the increases of the cases of $\mathrm{AD}$ among adult populations the paramount importance finds a single multitarget drug with an innovative therapeutic approach, in order to have a suitable treatment to help Alzheimer patients to improve their health conditions.

The Bolea investigations have not shown evidence on the selectivity of the enzymes toward this particular molecular set, their affect, and why significantly the activity is decreased when a hydrogen atom was attached to the nitrogen atom of the indole ring and/or when the side chain was substituted by a $\mathrm{CH}_{3}$ group [13]. However, the understanding of the factors that determine the exact nature of these interactions has not been found yet.

This study presents a theoretical investigation that could shed some light onto some molecular aspects that could contribute to the knowledge of the molecular mechanics behind interactions of these molecules with acetylcholinesterase $(\mathrm{AChE})$ and butyrylcholinesterase $(\mathrm{BuChE})$, as well as with monoamino oxidase (MAO) $A$ and $B$. All these issues 
TABLE 1: Structures and inhibitory activity of compounds analyzed.

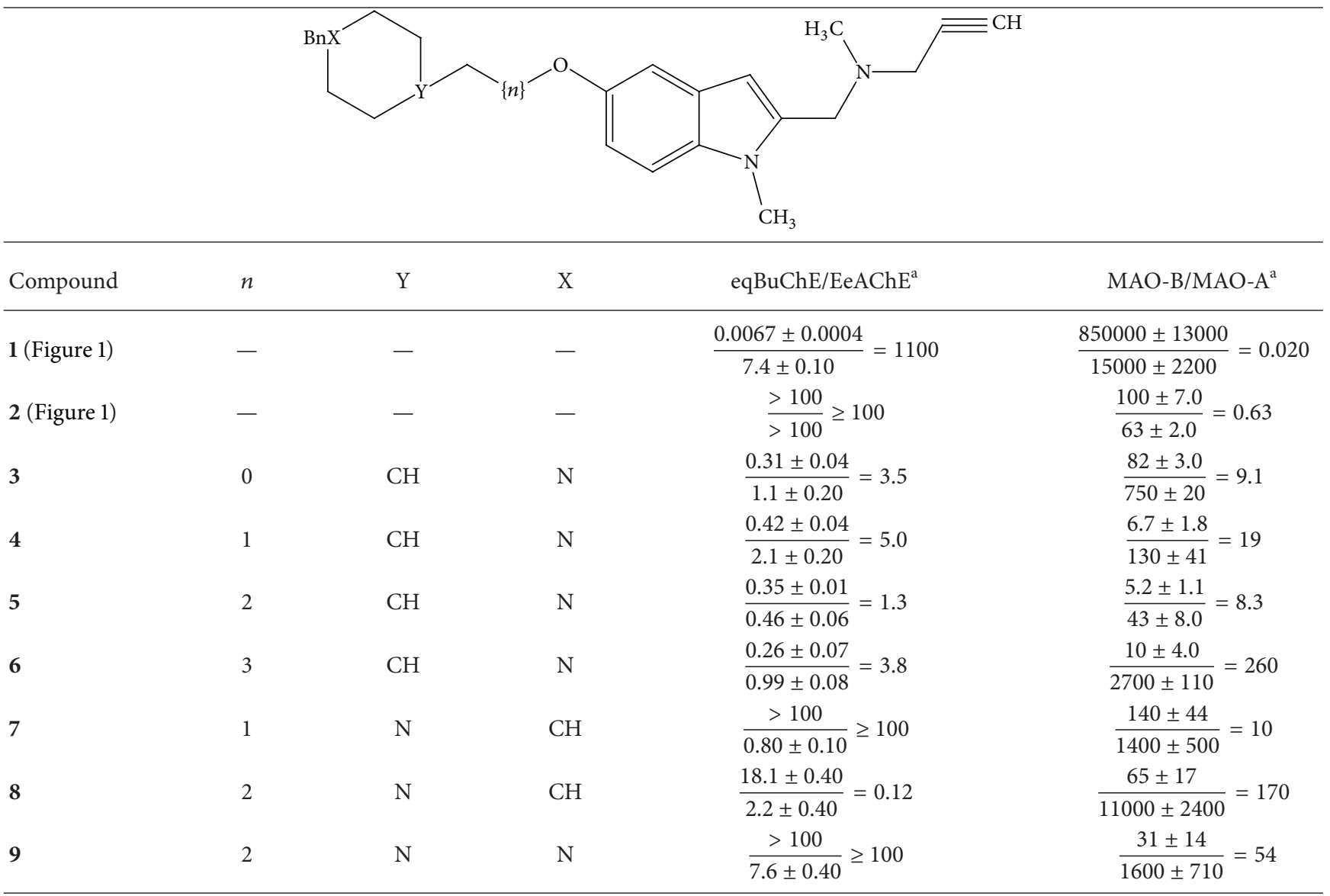

${ }^{\mathrm{a}} \mathrm{IC}_{50}(\mu \mathrm{M})$.

Note: values are expressed as the mean \pm standard error of the mean of at least three different experiments in quadruplicate [13].

prompted us to wonder whether these questions could be answered or, at least, clarified by theoretical approach. First, one can hope that theoretical descriptors based on quantum similarity (QS) $[17-24]$ and the Density Functional Theory (DFT) [25] can be used to get insights into the factors determining exact nature of the biological activity and selectivity of cholinesterase/monoamine oxidase inhibition and its interactions or at least trends, as to what factors affect the structural properties of these inhibitors molecules.

In this study the so-called response functions of the system are used to perturbations in the electrons number or external potential, commonly referred to as atomic or molecular reactivity descriptors. For instance, electronic chemical potential $(\mu)$, electrophilicity $(\omega)$, hardness $(\eta)$, electronegativity $(\chi)$, and softness $(S)$ are examples of such reactivity descriptors [26-30]. These reactivity indexes are used commonly to understand chemical reactivity and molecular selectivity [31-34]. These descriptors are supported on DFT [26-30]. In this work, the basic quantities to be addressed are hardness, electrophilicity, and chemical potential at the global level. A detailed discussion of these quantities can be found elsewhere (for detailed accounts see [26-30]). The local property developed in this work is the Fukui Function (FF). FF typically is a function that describes the local role of a specific atom within a molecule [31-34]. We also propose and seek a correlation and complementarity of reactivity descriptors with QS.

The similarity index concept was originally proposed from the perspective of quantum chemistry by Carbó and coworkers [17-24]. Similarity concepts is an omnipresent concept that permeates almost every chemistry field and other scientific fields such as physics, biology, and biochemistry among others. From the similarity or dissimilarity concept it is possible to obtain vital information of molecular designers, who are molecular designers within the drugdevelopment context, and the molecular similarity formalism has been proved to be one of the most significant computational tools that can be used to supply novel design ideas in order to get new drugs. The present work is concerned with such methodology that is based on similarity indexes computed from molecular fields using Carbó approach [1724].

Not long time ago the present authors have proposed a Quantitative Scales Convergence (QSC) [35, 36]. This study proposed quantitative concept of catecholamine allowing the definition of catecholamines as chemical group with the use of alpha and beta indexes which are used in the studies to quantify the transition state of the reaction paths 


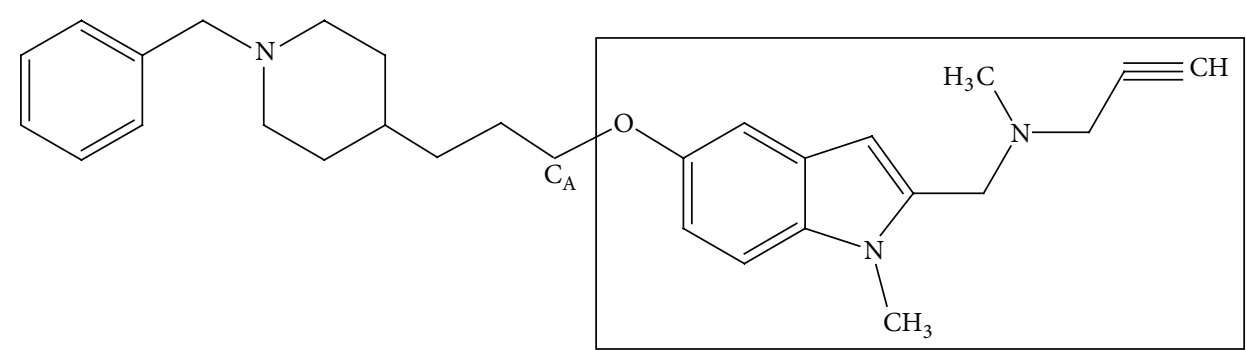

FIgURE 2: Structure of compound of the most biological activity (Compound 5, Table 1) which is used for the molecular alignment.

[37-39] and interpreted by the Cioslowski approach [40]. This study proposed a new interpretation of alpha and beta indexes to define the Overlap-QSC and Coulomb-QSC of dual cholinesterase/monoamine oxidase inhibitors for the Alzheimer Disease Treatment, reported by Bolea et al. [13]. In this sense, this study uses the alpha and beta indexes to quantify the hybrid nature of the compounds studied.

\section{Molecular Design, Geometry Optimization, and Molecular Descriptors Calculations}

The molecules analyzed and reported by Bolea and coworkers are shown in Table 1 [13]. These compounds were formed by hybridization of compounds $\mathbf{1}$ and $\mathbf{2}$ (see Figure 1).

2.1. Structures Alignment and Computational Details. In the similarity field the molecular arrangement or alignment of the molecules has played a central role, because the indexes values of molecular quantum similarity (MQS) are strongly dependent on the alignment method considered. Due of this dependence have been proposed by many alignment methods. Ranging from those used in methods CoMFA and CoMSIA which are in three dimensions (3D) and allow obtaining of steric maps, electrostatic maps, and hydrophobic maps, among others, [41, 42] these methods have been proposed in order to find alignment pattern that yields the best results by molecular alignment. In this study the Topogeometrical Superposition Algorithm alignment method has been used to handle flexible molecules (TGSA-Flex) [43]. This method is based on the comparison of the types of atoms and distances between them and the recognition of the largest common substructure in the aligned molecules. These types of search algorithms constitute an important field of scientific interest [44].

In this work, however, we decided to superimpose the common fragment shown in Figure 1, representing Compound $\mathbf{5}$ which is the most active molecules of this molecular set (Table 1); the common molecular fragment is highlighted in the structures of Table 1.

2.2. Computational Details. All molecular systems were optimized at the B3PW91 level [45-49] with the 6-31 G(d) [50] basis set using Gaussian 09 [51]. In addition the aminic nitrogen was not considered protonated, in order to study the binding interactions in neutral states in the context of the TGSA-Flex alignment; however the protonation effects can be associated from the computational point of view with molecular flexibility effects and in this sense we have applied the TGSA-Flex; this procedure is highly detailed and evaluated for set molecular: amino acids, nordihydroguaiaretic acid (NDGA) derivatives, HIV-1 protease inhibitors, and 1[2-hydroxyethoxy)methyl]-6-(phenylthio) thymine (HEPT) derivatives, among others [43]. In addition, the TGSA-Flex was evaluated by means of parameters as computational time, number of superposed atoms (also comparing it with respect to the rigid approach), and index of fit between the compared structures by Gironés and Carbó-Dorca [43]. Therefore in this study the TGSA-Flex has been used to postulate scales alpha and beta of quantitative convergence, according to Morales-Bayuelo et al. [35, 36].

2.3. Similarity Indexes. A significant problem in chemistry and biochemistry is to determine the similarity of two molecules $A$ and $B$. In order to measure the similarity between two molecules, Carbó-Dorca et al. [17, 24, 52] have introduced the concept of similarity index. They defined the QS measure $Z_{A B}$ between molecules $A$ and $B$ through the electronic density $\rho_{A}(r)$ (Compound 5, Figure 2) and $\rho_{B}(r)$ (molecular set) based on the idea of the minimizing of the expression for the distance as

$$
\begin{aligned}
D_{A B}^{2}= & \int\left|\rho_{A}(r)-\rho_{B}(r)\right|^{2} d r \\
= & \int \rho_{A}^{2}(r) d r+\int \rho_{B}^{2}(r) d r \\
& -2 \int \rho_{A}(r) \rho_{B}(r) d r \\
= & Z_{A A}+Z_{B B}-2 Z_{A B} .
\end{aligned}
$$

In this equation $Z_{A B}$ is the Overlap integral, often called molecular quantum similarity measure (MQSM), between the electronic densities of the molecules $A$ and $B . Z_{A A}$ and $Z_{B B}$ are called the molecular quantum self-similarity measures of molecules $A$ and $B$ [53]. In the (MQS) field, densities functions are considered as vectors and can be defined mathematically as

$$
R_{A B}=\frac{\int \rho_{A}(r) \rho_{B}(r) d r}{\left[\left(\int \rho_{A}^{2}(r) d r\right)\left(\int \rho_{B}^{2}(r) d r\right)\right]^{1 / 2}}=\frac{Z_{A B}}{\sqrt{Z_{A A} Z_{B B}}} .
$$

The Carbó index is limited to the range $(0,1]$, where $C_{A B}=1$ means perfect similarity. The range of the 
Carbó index naturally agrees with the Schwartz integral inequality [54]

$$
\left[\int \rho_{A}(r) \rho_{B}(r) d r\right]^{2} \leq \int \rho_{A}^{2}(r) d r \int \rho_{B}^{2}(r) d r
$$
(2).

When $A$ equals $B$, the similarity measure $\left(Z_{A B}\right)$ in the

\subsubsection{Local Similarity Indexes (LSI)}

The Hirshfeld Approach. In this study the use of the Hirshfeld approach for the quantification of Hybrid character IMAO/IAChe is postulated [13]. The Hirshfeld method was developed [55], for the calculation of stockholders charge partitioning, and is a method widely used in the DFT [56, 57]; this Hirshfeld approach in this study is used taking in account the holographic electron density theorem postulated by Mezey [58].

This Hirshfeld approach is based on the partioning of the electronic density $\rho(r)$ of a molecule in contributions $\rho_{A}(r)$. These contributions are proportional to the weight $w_{A}(r)$ of the electronic density of the isolated molecule in the call promolecular density $[59,60]$ This weight is defined as the ratio of the electronic density of the isolated atom constructed from the superposition of the densities of all atoms isolated in the same position of the molecule ("the promolecular density"); this is obtained as

$$
\rho_{A}^{\text {Prom }}(r)=\sum_{x} \rho_{x}^{0}(r)
$$

The contribution of atom $A$ (see Figure 2), $\rho_{A}(r)$ the electronic density $\rho(r)$ is obtained as

$$
\rho_{A}(r)=w_{A}(r) \rho(r)
$$

whereby the weight $\left(w_{A}(r)\right)$ is obtained as

$$
w_{A}(r)=\frac{\rho_{A}^{0}}{\sum_{x} \rho_{x}^{0}(r)}
$$

where $\rho_{A}^{0}(r)$ is the electronic density of the isolated atom $A$. Recently, the group Carbó-Dorca used the Hirshfeld partition to be able to express the overall (QS) index equation 2 at local level $[59,60]$.

The contribution of Carbon atom $\left(C_{A}\right)$ in the molecule $A$ is given by

$$
\rho_{C_{A}, A}(r)=w_{C_{A}}(r) \rho_{A}(r)
$$

with

$$
w_{C_{A}, A}=\frac{\rho_{C_{A}, A}^{0}(r)}{\sum_{x} \rho_{x}^{0}(r)} .
$$

Equally to it is the contribution atomic of Carbon atom $\left(C_{B}\right)$ in the molecule $B$ (set molecular, Figure 1 ) which is obtained as

with

$$
\rho_{C_{B}, B}(r)=w_{C_{B}}(r) \rho_{B}(r)
$$

$$
w_{C_{B}, B}=\frac{\rho_{C_{B}, B}^{0}(r)}{\sum_{x} \rho_{x}^{0}(r)} .
$$

So we can write the contribution of the asymmetric Carbon atom $\left(C_{A}+C_{B}: C\right)$ products $\rho_{A}(r) \rho_{B}(r)$ as

$$
\rho_{C: A, B}(r)=w_{C: A, B}(r) \rho_{A}(r) \rho_{B}(r),
$$

where

$$
Z^{\text {local }, C_{A, B}}=\int w_{C} \rho(r) d r=\int\left(\frac{\rho_{C_{A, B}}^{0}(r)}{\sum_{X} \rho_{X, C}^{0}(r)}\right) \rho_{C_{A}}(r) d r
$$

where $\sum_{x} \rho_{x, C}^{0}(r)$ is the total promolecular density overlay of the two molecules considered, so that we can express the numerator $Z_{A B}$ Carbó index (2) as

$$
\begin{aligned}
R_{C: A, B}^{\text {local }} & =\frac{Z^{\text {local }, C_{A}} Z^{\text {local }, C_{B}}}{\sqrt{\left(Z^{\text {local }, C_{A}}\right)^{2}\left(Z^{\text {local }, C_{B}}\right)^{2}}} \\
& =\frac{\int\left(\rho_{C_{A}}^{0}(r) / \sum_{X} \rho_{X, C_{A}}^{0}(r)\right)\left(\rho_{C_{B}}^{0}(r) / \sum_{Y} \rho_{Y, C}^{0}(r)\right) \rho_{C_{A}}(r) \rho_{C_{B}}(r) d r}{\left[\int\left(\rho_{C_{A}}^{0}(r) / \sum_{X} \rho_{X, C_{A}}^{0}(r)\right)^{2} \rho_{C_{A}}(r) \rho_{C_{A}}(r) d r\right]^{1 / 2}\left[\int\left(\rho_{C_{B}}^{0}(r) / \sum_{Y} \rho_{Y, C_{B}}^{0}(r)\right)^{2} \rho_{C_{B}}(r) \rho_{C_{B}}(r) d r\right]^{1 / 2}}
\end{aligned}
$$

The global index is developed in (2). In addition the Hirshfeld approach is deeply connected to molecular similarity as was stressed in the recent work of Mezey [58].
Alpha and Beta Indexes Proposed. In order to create the (QSC) to quantify the nature of hybridization of IMAO/IAChe, Figure 1 [13] the alpha and beta indexes proposed by 
Cioslowski were used [40] and interpreted by us $[35,36]$ which allows postulation of two scales which define the (QSC). The alpha index is defined mathematically as

$$
\alpha=\frac{Z_{A C}^{x}+Z_{C B}^{x}}{Z_{A B}^{x}},
$$

where $Z_{A B}^{x}$, when $x=\mathrm{OI}$, represent the molecular quantum Overlap Index (OI), $x=\mathrm{CI}$ represent the molecular quantum Coulomb Index (CI), and $Z_{A B}$ is the index (15) that has a range defined by the interval $(0,2)$; the values of these indexes are determined by $\lim _{Z_{A B}^{x} \rightarrow 0} \alpha$, when alpha $(\alpha)$ takes a value of zero which means that there is dissimilarity between $A$ and $C$, and $C$ with $B$ and their self-similarity between the molecules $A$ (Compound 1 ) and $B$ (Compound 2 ), taking the value of 2 is because $A=B=C$. We interpret this index as an index of correlation group, which allows us to justify the inhibitors character in this study. In (15), the molecule $A$ represents compound 1, molecule $B$ represent compound 2 , and molecule $C$ is the molecular set.

The second index used here [37] was the beta index which allows considerations of distances. This index is mathematically defined as

$$
\beta=\frac{D_{A C}^{x}-D_{C B}^{x}}{D_{A B}^{x}},
$$

where $D_{A B}^{x}$, when $x=0$ is the distance of Overlap, for $x=\mathrm{C}$ is the Distance of Coulomb; this has a range of convergence $(-1,1)$, when taking a negative value means that the molecule $A$ is more similar to the molecule $C$ with respect to the molecule $B$, when taking the value of 1 means that the molecule $B$ is more similar to the molecule $C$, with respect to the molecule $A$, taking into account that the decrease in the distance increases the (QS).

2.4. Global and Local Reactivity Descriptors. As a detailed presentation and discussion of the DFT based descriptors of the reactivity used in this paper can be found elsewhere [26-30, 61-69], only the relevant expressions used for the evaluation of different quantities for hybrid compounds are given here. The global reactivity indexes can be obtained as

$$
\begin{gathered}
\mu \approx \frac{\varepsilon_{L}+\varepsilon_{H}}{2}, \\
\eta \approx \frac{\varepsilon_{L}-\varepsilon_{H}}{2}, \\
S=\frac{1}{\eta},
\end{gathered}
$$

where $\mu$ is the chemical potential, $\eta$ is global hardness, and $S$ is the global softness. $\varepsilon_{L}$ and $\varepsilon_{H}$ represent the energies of the highest occupied molecular orbital (HOMO) and lowest unoccupied molecular orbital (LUMO), respectively.

Using (16) and (17) it is possible determine the electrophilicity $(\omega)$ index, defined as

$$
\omega=\frac{\mu^{2}}{2 \eta}
$$

where $\omega$ is another global property and represents the stabilization energy of the system when it is saturated by electrons from the external environment.

The descriptors expressed in (16)-(19) also are known as global system descriptors and depend on $N$ and $\nu(r)$ and they provide information on the reactivity and stability of a chemical system [26-30, 59-69]. Local properties are obtained taking into account the variation of energy with respect to the external potential; this variations depend on the position $(r)$ and defined as a selectivity index.

However, in order to study selectivity and reactivity it is more advisable to use local reactivity descriptors. Within these we have the FF [31-34, 70, 71] which explain the selectivity of a region of a molecule and defined mathematically as

$$
\langle f(\vec{r})\rangle=\left(\frac{\delta \mu}{\delta \nu(\vec{r})}\right)_{N}=\left(\frac{\partial \rho(\vec{r})}{\partial N}\right)_{\nu(\vec{r})}=\left(\frac{\partial^{2} E}{\partial N \partial v(\vec{r})}\right) .
$$

The FF is interpreted as the change of chemical potential given an external perturbation, or the variation of the electronic density function when the electrons number change. For the calculation of the FF $\left(f^{+}\right)$we used the condensed approach proposed by Yang and Mortier [72], for the systems with $N$ and $N+1$ electrons:

$$
\begin{gathered}
\left\langle f_{k}^{+}(\vec{r})\right\rangle=\int_{k}\left[\rho_{N+1}(\vec{r})-\rho(\vec{r})\right] d r, \\
\left\langle f_{k}^{-}(\vec{r})\right\rangle=\int_{k}\left[\rho_{N}(\vec{r})-\rho_{N-1}(\vec{r})\right] d r, \\
\left\langle f_{k}^{0}(\vec{r})\right\rangle=\frac{1}{2}\left[\left\langle f_{k}^{+}\right\rangle+\left\langle f_{k}^{-}\right\rangle\right]
\end{gathered}
$$

with $q_{k}(N)$ denoting the electronic population of atom $k$ of the system under study; these FF in the condensate orbital approximation model consider only the contribution of the frontier orbitals of an atom [73]:

$$
\begin{gathered}
\left\langle f_{k}^{+}\right\rangle=\int_{k} \rho_{L}(\vec{r}) d \vec{r}, \\
\left\langle f_{k}^{-}\right\rangle=\int_{k} \rho_{H}(\vec{r}) d \vec{r}, \\
\left\langle f_{k}^{0}\right\rangle=\frac{1}{2}\left[\left\langle f_{k}^{+}\right\rangle+\left\langle f_{k}^{-}\right\rangle\right]
\end{gathered}
$$

representing the electronic populations on atom $k$ of the LUMO and HOMO orbitals, respectively.

\section{Results and Discussion}

In the Tables 2-5 the MQS indexes calculated with (14) are shown, in order to quantify the nature of hybridization IMAO/IAChe [13-16], proposed in this study.

The higher Overlap index values found in Table 2 are between the molecules 8 and 9 with a value of 0.8812 , with a distance value of 2.4539 (Table 3 ). The lowest value using the Overlap index of similarity is obtained between the molecules 9 and 1 with a value of 0.4117 , with a distance of 5.3077 , in agreement with the experimental results (see Table 1). 
TABLE 2: Molecular quantum similarity matrix using the Overlap operator.

\begin{tabular}{lcccccccc}
\hline $\mathrm{C}^{\mathrm{a}}$ & 1 & 2 & 3 & 4 & 5 & 6 & 7 & 8 \\
\hline $\mathbf{1}$ & 1.0000 & & & & & & & \\
$\mathbf{2}$ & 0.5276 & 1.0000 & & & & & & \\
$\mathbf{3}$ & 0.4518 & 0.5665 & 1.0000 & & & & \\
$\mathbf{4}$ & 0.4250 & 0.6146 & 0.6188 & 1.0000 & & & \\
$\mathbf{5}$ & 0.4237 & 0.6222 & 0.5889 & 0.6080 & 1.0000 & & & \\
$\mathbf{6}$ & 0.4119 & 0.6103 & 0.5793 & 0.5981 & 0.7291 & 1.0000 & & \\
$\mathbf{7}$ & 0.4314 & 0.6218 & 0.6795 & 0.7138 & 0.6082 & 0.6008 & 1.0000 & \\
$\mathbf{8}$ & 0.4159 & 0.6253 & 0.5845 & 0.6035 & 0.6488 & 0.6440 & 0.6082 & 1.0000 \\
$\mathbf{9}$ & 0.4117 & 0.6204 & 0.5790 & 0.5969 & 0.6436 & 0.6359 & 0.6028 & 0.8812 \\
\hline
\end{tabular}

${ }^{\mathrm{a}} \mathrm{C}$ : Compound (Table 1).

TABLE 3: Molecular quantum similarity matrix using the Overlap distances.

\begin{tabular}{lcccccccc}
\hline $\mathrm{C}^{\mathrm{a}}$ & 1 & 2 & 3 & 4 & 5 & 6 & 7 & 8 \\
\hline $\mathbf{1}$ & 0.0000 & & & & & & & \\
$\mathbf{2}$ & 4.3502 & 0.0000 & & & & & & \\
$\mathbf{3}$ & 5.0081 & 4.2452 & 0.0000 & & & & \\
$\mathbf{4}$ & 5.1784 & 3.9566 & 4.2717 & 0.0000 & & & & \\
$\mathbf{5}$ & 5.2258 & 4.0638 & 4.4708 & 4.4029 & 0.0000 & & & \\
$\mathbf{6}$ & 5.3214 & 4.1679 & 4.5580 & 4.4916 & 3.7135 & 0.0000 & & \\
$\mathbf{7}$ & 5.1082 & 3.9802 & 3.8866 & 3.7066 & 4.3702 & 4.4458 & 0.0000 & \\
$\mathbf{8}$ & 5.2610 & 4.0475 & 4.4947 & 4.4279 & 4.1975 & 4.2573 & 4.3706 & 0.0000 \\
$\mathbf{9}$ & 5.3077 & 4.1015 & 4.5481 & 4.4874 & 4.2496 & 4.3254 & 4.4233 & 2.4539 \\
\hline
\end{tabular}

${ }^{\mathrm{a}} \mathrm{C}$ : Compound (Table 1).

TABLE 4: Molecular quantum similarity matrix using the Coulomb operator.

\begin{tabular}{lcccccccc}
\hline $\mathrm{C}^{\mathrm{a}}$ & 1 & 2 & 3 & 4 & 5 & 6 & 7 & 8 \\
\hline $\mathbf{1}$ & 1.0000 & & & & & & & \\
$\mathbf{2}$ & 0.8435 & 1.0000 & & & & & & \\
$\mathbf{3}$ & 0.7634 & 0.8852 & 1.0000 & & & & \\
$\mathbf{4}$ & 0.7317 & 0.8748 & 0.9013 & 1.0000 & & & & \\
$\mathbf{5}$ & 0.7131 & 0.8634 & 0.8376 & 0.8320 & 1.0000 & & & \\
$\mathbf{6}$ & 0.7154 & 0.8470 & 0.8178 & 0.8273 & 0.9124 & 1.0000 & & \\
$\mathbf{7}$ & 0.7471 & 0.8782 & 0.9245 & 0.9384 & 0.8146 & 0.8045 & 1.0000 & \\
$\mathbf{8}$ & 0.7585 & 0.8768 & 0.8262 & 0.8217 & 0.8451 & 0.8450 & 0.8160 & 1.0000 \\
$\mathbf{9}$ & 0.7543 & 0.8746 & 0.8241 & 0.8211 & 0.8435 & 0.8459 & 0.8159 & 0.9896 \\
\hline
\end{tabular}

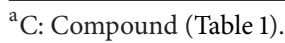

TABLE 5: Molecular quantum similarity matrix using the Coulomb distances.

\begin{tabular}{|c|c|c|c|c|c|c|c|c|c|}
\hline $\mathrm{C}^{\mathrm{a}}$ & 1 & 2 & 3 & 4 & 5 & 6 & 7 & 8 & 9 \\
\hline 1 & 0.0000 & & & & & & & & \\
\hline 2 & 28.4575 & 0.0000 & & & & & & & \\
\hline 3 & 38.1561 & 27.1602 & 0.0000 & & & & & & \\
\hline 4 & 40.9282 & 28.5893 & 25.6404 & 0.0000 & & & & & \\
\hline 5 & 43.1555 & 30.7357 & 33.4469 & 34.1829 & 0.0000 & & & & \\
\hline 6 & 43.3228 & 32.5861 & 35.6565 & 34.8799 & 25.1628 & 0.0000 & & & \\
\hline 7 & 39.2375 & 27.5623 & 22.1910 & 20.1991 & 35.6274 & 36.8308 & 0.0000 & & \\
\hline 8 & 39.9759 & 29.9113 & 34.8076 & 35.4144 & 33.4286 & 33.6160 & 35.7177 & 0.0000 & \\
\hline 9 & 40.3314 & 30.1456 & 35.0363 & 35.4929 & 33.6203 & 33.5376 & 35.8290 & 8.7038 & 0.0000 \\
\hline
\end{tabular}

${ }^{\mathrm{a} C}$ : Compound (Table 1). 
TABLE 6: Alpha and beta indexes proposed in this study, using (15)-(16).

\begin{tabular}{lcccc}
\hline Compound & $\alpha^{\mathrm{O}}$ & $\alpha^{\mathrm{C}}$ & $\beta^{\mathrm{O}}$ & $\beta^{\mathrm{C}}$ \\
\hline $\mathbf{3}$ & 1.9303 & 1.9544 & -0.1754 & -0.3864 \\
$\mathbf{4}$ & 1.9704 & 1.9046 & -0.2809 & -0.4336 \\
$\mathbf{5}$ & 1.9824 & 1.8690 & -0.2671 & -0.4364 \\
$\mathbf{6}$ & 1.9375 & 1.8523 & -0.2651 & -0.3773 \\
$\mathbf{7}$ & 1.9963 & 1.9269 & -0.2593 & -0.4362 \\
$\mathbf{8}$ & 1.9736 & 1.9388 & -0.2789 & -0.3537 \\
$\mathbf{9}$ & 1.9567 & 1.9312 & -0.2932 & -0.3579 \\
\hline
\end{tabular}

The most similar compound with respect to 5 (more active of set) is the compound $\mathbf{6}$ with an Overlap index value between them of 0.7291 and a distance of 3.7135. The methylene group additional to the compound 6 with respect to 5 increased the inhibitory potency against MAO- $A$ and MAO- $B$ by a factor of 2 and 63, respectively. Despite of the high similarity between these two molecules, they have different hybridization character as is depicted in Table 1. The compound 5 has a selectivity eq BuChE/EeAChE value of $1.3 \mu \mathrm{M}$ whereas the compound $\mathbf{6}$ has a selectivity value of $3.8 \mu \mathrm{M}$. Also, there is a more drastic contrast between these molecules, when the selectivity values of MAO-B/MAO- $A$ are compared: $8.3 \mu \mathrm{M}$ for molecule 5 and a value of $260 \mu \mathrm{M}$ for molecule 6 . In order to characterize the hybrid character of this molecular set from electronic point of view the index of Coulomb similarity was calculated (see Tables 4 and 5).

The higher Coulomb index value is found between the molecules 8 and 9 with a value of 0.9896 (see Table 4) despite of their differences in eqBuChE/EeACh and MAO-B/MAO$A$ inhibition with a distance of 8.7038 (Table 5). The lowest value is found between molecules 5 and 1 (Table 4) with a value of 0.7131 and a distance of 43.1555 .

The similarity values (Tables 2-5) are used to calculate the alpha and beta indexes $[35,36]$. The higher alphaOverlap $\left(\alpha^{\mathrm{O}}\right)$ index value was 1.9963 to molecule 7. The lowest value found for alpha-Overlap $\left(\alpha^{\mathrm{O}}\right)$ index was 1.9303 for compound 3. The $\alpha^{\mathrm{O}}$ index is associated with the structural similarity of the compound of interest with respect to the compounds 1 and 2, respectively, which are responsible for the hybrid character. The higher value found for alphaCoulomb $\left(\alpha^{\mathrm{C}}\right)$ index was 1.9544 . The lowest value found for alpha-Coulomb $\left(\alpha^{\mathrm{C}}\right)$ is 1.8523 to Compound 6. The alpha-Coulomb $\left(\alpha^{\mathrm{C}}\right)$ index is associated with the electronic similarity of the compound of interest with respect to the compounds 1 and 2, respectively.

Compound 5 has an alpha-Overlap $\left(\alpha^{\mathrm{O}}\right)$ index of 1.9824 and alpha-Coulomb $\left(\alpha^{\mathrm{C}}\right)$ index of 1.8690. Taking into account that these similarity indexes values can be related with hybrid character of the molecules with some steric considerations determinated by electronic effects in the binding site, these values are consistent with the $\beta^{\mathrm{O}}:-0.2671$ and $\beta^{\mathrm{C}}:-0.4364$ values (see Table 6 ). $\beta$ Index is associated with the distances of Overlap and Coulomb, respectively, of the molecular set with respect to the compounds $\mathbf{1}$ and $\mathbf{2}$. In Figure 3 the values obtained using these (QSC) are shown .

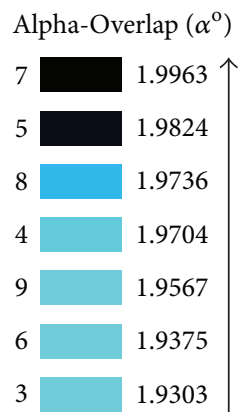

(a)

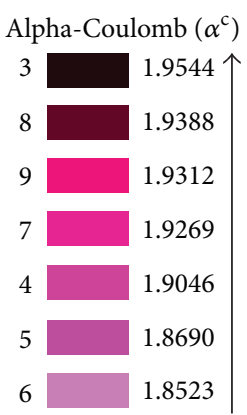

(b)
FIGURE 3: Overlap and Coulomb Quantitative Convergence Scales, respectively (see (15) and (16)).

Note, however, that the order obtained in both scales is not the same. In Figure 3 the molecule 3 has the lowest value of alpha-Overlap $\left(\alpha^{\mathrm{O}}\right)$, whereas the molecule 3 has (Figure 3 ) the higher value in the alpha-Coulomb scales $\left(\alpha^{C}\right)$. This denotes a possible dual inhibition from electronic perspective. The relationships found in this work allow quantifying the hybrid character of IMAO/IAChe (Figure 4) [13].

As a result of invariance (self-similarity) of the system, we can define scales according to Barrow [74]. In this sense, the biological activity can be interpreted in terms of calculated self-similarity and related with hybrid character of these inhibitors. Taking into account that Coulomb index has the higher values, electronic properties of these molecules are analyzed from the chemical reactivity perspective, showing that the biological activity can be estimated using the alpha and beta indexes proposed (Table 7).

The chemical potential sequence outlined in Table 7 yields $1(\mu=-3.9031)>2(\mu=-2.6169)>7(-2.5085)>$ $6(-2.5021)>4(\mu=-2.4981)>8(\mu=-2.4945)>5(\mu=$ $-2.4937)>9(\mu=2.4916)>3(\mu=-2.2839)$. Whereas the electrophilicity tendency is $1(\omega=2.3113)>2(\omega=0.7006)>$ $7(\omega=0.6432)>6(\omega=0.6378)>4(\omega=0.6362)>8(\omega=$ $0.6341)>5(\omega=0.6334)>9(\omega=0.6329)>1(\omega=0.6001)$. The chemical potential analysis and electrophilicity trend leads to some general comments. At first glance, these two sequences follow the same trend. The electrophilicity index represents the stabilization energy of the system when it is saturated by electrons from the external environment. On the other hand, the hardness values to molecule $5(\eta: 4.9084 \mathrm{eV})$ 


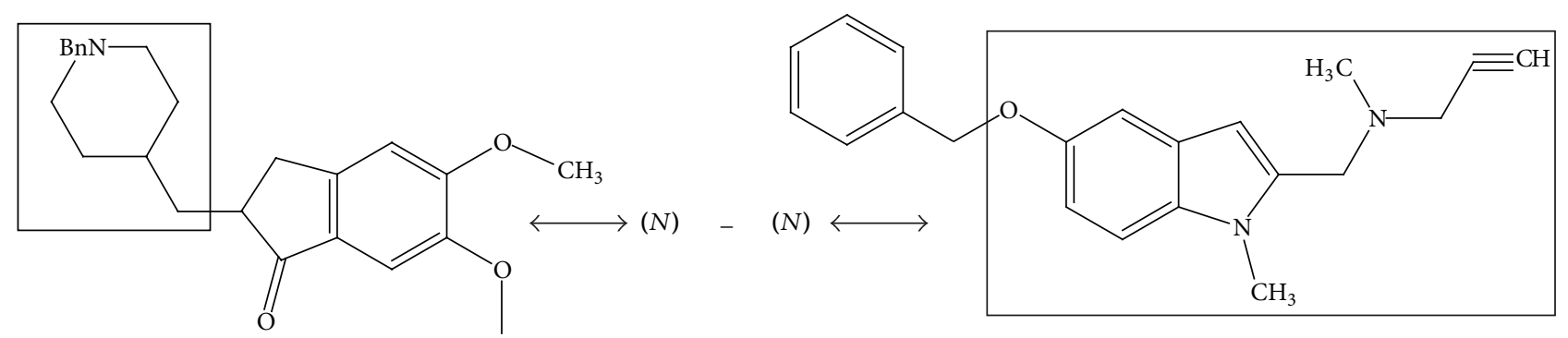

Donepezil (1)

(2)

$\left(\beta^{X}\right)=$

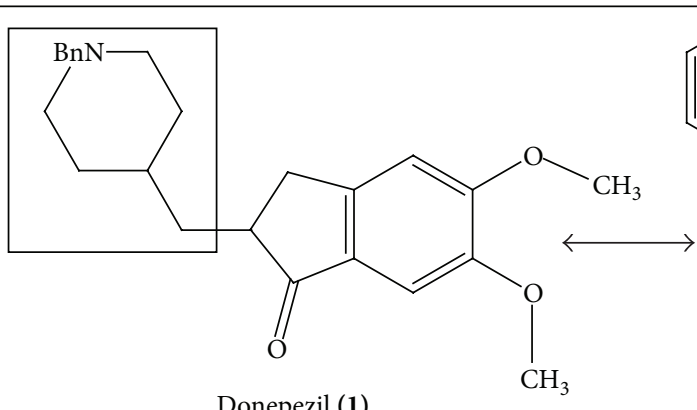

Donepezil (1)

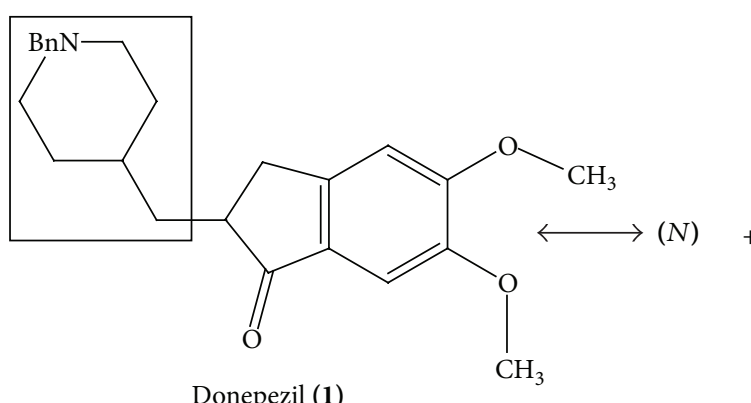

Donepezil (1)

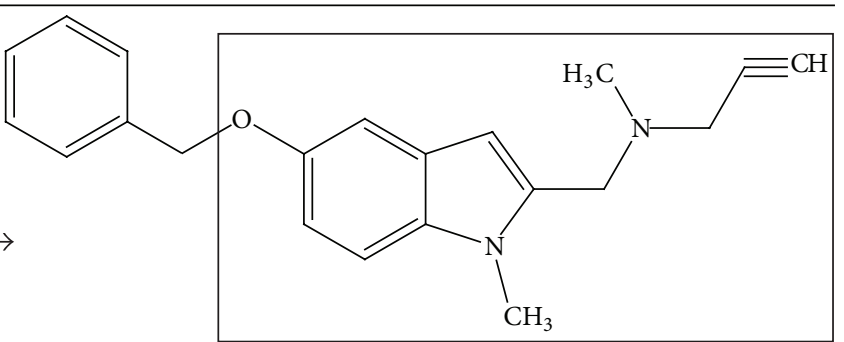

(2)

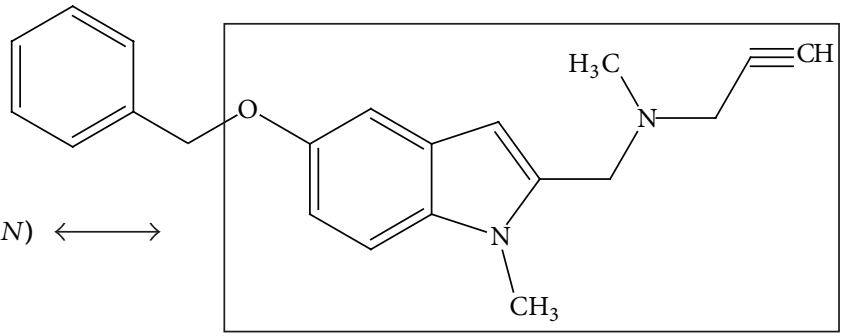

(2)

$\left(\alpha^{X}\right)=$<smiles>C=CCCC1Cc2cc(OC)c(OC)cc2C1=O</smiles>

Donepezil (1)

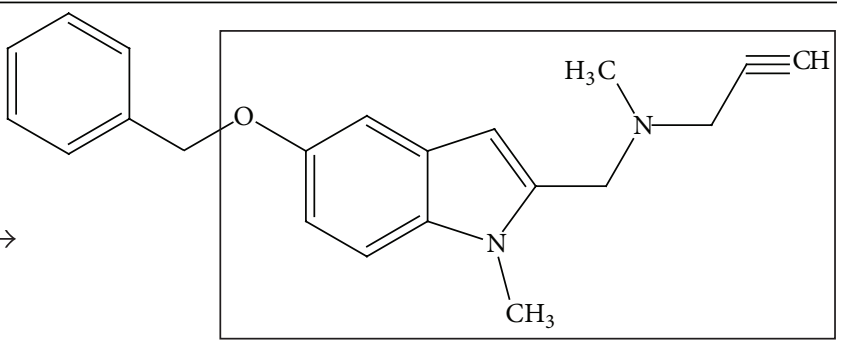

(2)

Figure 4: Shows the relationships that define the alpha and beta indexes proposed in this study. Note: $X$ : Overlap index or Coulomb index, $N$ : Molecular set.

show that this compound is very stable, despite of their high inhibition activity (see Table 1); this can be rationalized with a high selectivity to eq BuChE/EeACh and moderate inhibition with respect to MAO.

The FF turns out to be essential for estimating relative interaction of different sites in a molecule towards electrophilic or nucleophilic reagents. The FF can provide a firm explanation of the reactivity of these molecular set to characterize the reactivity sites from the local point of view.
The higher $\mathrm{FF}\left(F^{+}\right)$value represents the probability for a nucleophilic attack (Figure 5(b)). The FF $\left(F^{-}\right)$values in the compound $\mathbf{5}$ indicate that the central ring of this molecule in special carbon $3\left(\mathrm{C}_{3}\right)$ is the most susceptible to be attacked by electrophilic species, whereas the ring of five members of the same molecule is most susceptible to be attacked by nucleophilic species. This means that the AChE inhibition and MAO $B$ is due to retrodonation on the central ring that allows the dual inhibition on cholinesterase. The MAO 


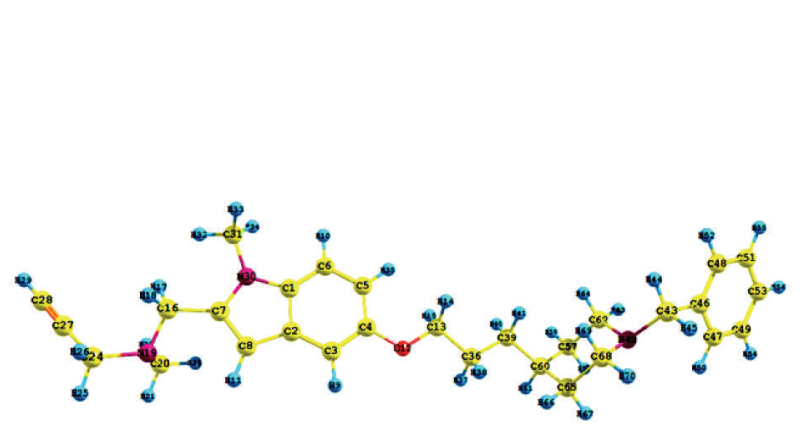

(a)

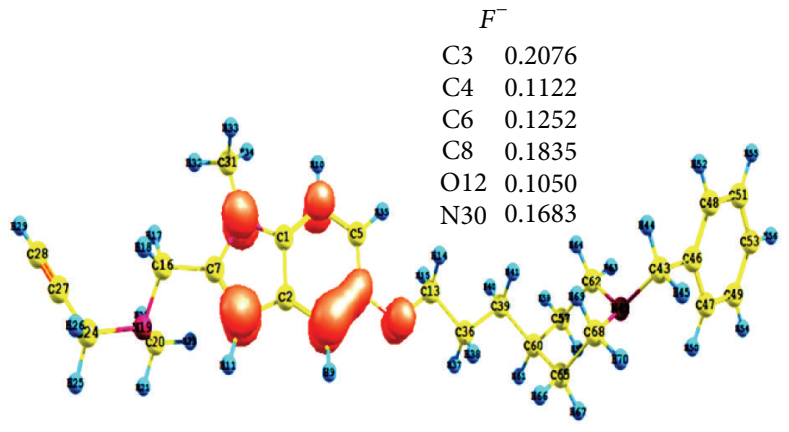

(b)

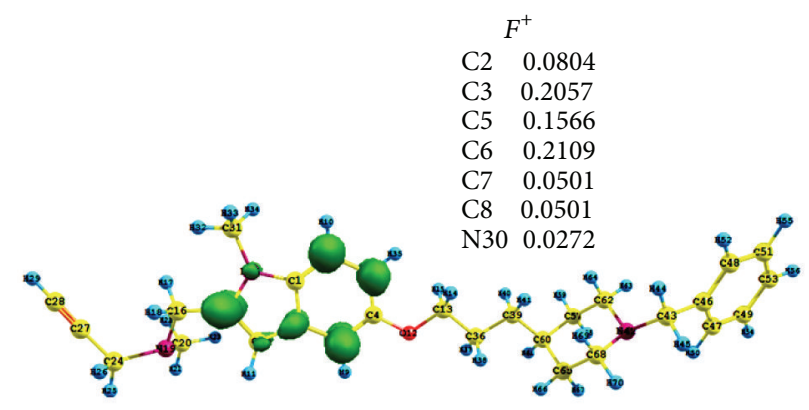

(c)

Figure 5: (a) Surfaces 0.005 of the Fukui Functions, to Compound 5, (b) Region $F^{-}(r)$ for electrophilic attack in orange $y$, and (c) Region $F^{+}(r)$ for nucleophilic attack in green.

TABLE 7: Global reactivity indexes, for cholinesterase/monoamine oxidase inhibitors.

\begin{tabular}{lccc}
\hline Compound & $\mu^{\mathrm{a}}(\mathrm{eV})$ & $\eta^{\mathrm{b}}(\mathrm{eV})$ & $\omega^{\mathrm{c}}(\mathrm{eV})$ \\
\hline $\mathbf{1}$ & -3.9031 & 3.2956 & 2.3113 \\
$\mathbf{2}$ & -2.6169 & 4.8877 & 0.7006 \\
$\mathbf{3}$ & -2.2839 & 4.3457 & 0.6001 \\
$\mathbf{4}$ & -2.4981 & 4.9049 & 0.6362 \\
$\mathbf{5}$ & -2.4937 & 4.9084 & 0.6334 \\
$\mathbf{6}$ & -2.5021 & 4.9079 & 0.6378 \\
$\mathbf{7}$ & -2.5085 & 4.9136 & 0.6432 \\
$\mathbf{8}$ & -2.4945 & 4.9062 & 0.6341 \\
$\mathbf{9}$ & -2.4916 & 4.9043 & 0.6329 \\
\hline
\end{tabular}

${ }^{\mathrm{a}}$ Chemical potential.

${ }^{\mathrm{b}}$ Hardness.

${ }^{\mathrm{c}}$ Electrophilicity.

inhibition is determinated by steric and electronic effect. These can be characterized from the chemical point of view with the binding sites of inhibition and selectivity.

In Figure 5 we can see that the retrodonor process is due to the $\mathrm{C} 3, \mathrm{C} 4, \mathrm{C} 6, \mathrm{C} 8, \mathrm{O} 12$, and $\mathrm{N} 30$ atoms for regions $\left(F^{-}\right.$) (Figure 5(a)) and $\mathrm{C} 2, \mathrm{C} 3, \mathrm{C} 5, \mathrm{C} 6, \mathrm{C} 7, \mathrm{C} 8$, and $\mathrm{N} 30$ atoms for regions $\left(F^{+}\right)$(Figure $5(\mathrm{~b})$ ) for the more active molecule 5 that causes one electronic donation capacity quantified by the electrophilicity index (see Table 7) which allows the stabilization in the active site and postulate the retrodonor process by the $\mathrm{C} 3, \mathrm{C} 6, \mathrm{C} 8$, and $\mathrm{CN} 30$ (Figure 5(a)) atoms. However the molecular set studied is hybrid molecules that act as MAO- $B$ inhibitors by virtue of the covalent bond formed by the propargylamino moiety with the Flavin Adenine Dinucleotide (FAD) cofactor and as ChE inhibitors by interaction at the catalytic site with the N-benzyl-cycloalkylamino moiety and at the peripheral site (PAS of AChE) with the heteroaromatic moiety [13, 75-81]. Therefore, retrodonation on the central ring might even take place but as an additional interaction.

Due to the hybrid character of the inhibitors studied determined by the combination of pharmacological and neuroprotective activities in a same molecule, by part of the cholinesterase inhibition is inhibits the cholinesterase preventing that destroy the acetylcholine released, producing as a consequence an increase in the concentration and duration of the effects of neurotransmitter and on the other hand blocking the action of the MAO enzyme [52]. The description from the point of view of mechanical inhibition requires a correct interpretation of the structures involved in the process, to postulate new methodologies as the one developed in this study to quantify the character inhibitor dual is of paramount importance (see Figure 4); in addition from the point of view of the chemical reactivity the interactions in the active site by retrodonor process quantified by the FF are shown which may be useful for design of new drugs with better selectivity, molecular recognition, and pharmacological activity for the AD [13-16, 82, 83]. 


\section{Conclusions}

From the whole of the results presented in this contribution, it can be concluded that through the use of MQS indexes such as Coulomb, Overlap, and their respective distances it is possible to quantify the electronic and steric effect that gives rise to QSC. These scales permit the stratification of the steric and electronic character. The main output of the similarity indexes alpha-Overlap $\left(\alpha^{\mathrm{O}}\right)$ and alpha-Coulomb $\left(\alpha^{\mathrm{C}}\right)$ calculations can be related with hybrid character of the molecules and with some steric considerations on the binding site, providing a tool to semiempirical quantum chemistry group that provides a clear correlation, defining groups of compounds isolated from a different way than has been done in the chemical literature (chemical series and functions), Figures 3 and 4.

Topogeometrical Superposition Algorithm to handle flexible molecules (TGSA-Flex) was used as alignment method. This method provides a straightforward procedure to solve the problem of molecular relative orientation taking into account that the inhibitors considering can be protonated or deprotonated. It provides us with a tool to evaluate MQS and permitting association of the protonation process with molecular flexibility effects.

In fact, the interaction strength pattern of these molecules can be explained by the use of a series of reactivity descriptors such as chemical potential, electrophilicity, and Fukui Function. Moreover, a quantitative agreement of DFT calculations and expression derived has been put forward and provided insight into the ordering of the chemical potential and electrophilicity of the Bolea molecular set.

The Fukui outcome provided insight into the possible interaction of these molecules and gives explications about the AChE and MAO B inhibition that can be considered in terms of retrodonation interactions on the central ring, which helps the dual inhibition of cholinesterase. These mean that the MAO inhibition can be studied by steric and electronic effect. For example in the case of molecule 5 the FF show that the central ring of this molecule is favorable to nucleophilic and electrophilic attack postulating the retrodonation process on the central ring as an additional interaction in the stabilization of central site, providing new considerations about the bonding processes in such inhibitors. These properties obviously are modulated by their influence in the type of interactions that these molecules can undergo.

\section{Acknowledgments}

Ricardo Vivas-Reyes wishes to thank the Universidad de Cartagena (Cartagena de Indias, Colombia), for continuous support to his group. Alejandro Morales-Bayuelo thank the Universidad Nacional Andres Bello (Santiago-Chile), for a Ph.D. fellowship: CONICYT (63100003). Finally the authors thank their friend Ramon Carbó-Dorca (Universitat of Girona, Spain) for the TGSA-Flex [43].

\section{References}

[1] M. Goedert and M. G. Spillantini, "A century ofAlzheimer's disease," Science, vol. 314, pp. 777-781, 2006.
[2] C. Berchtold and C. W. Cotman, "Evolution in the conceptualization of Dementia and Alzheimer's disease: greco-roman period to the 1960s," Neurobiology of Aging, vol. 19, no. 3, pp. 173-189, 1998.

[3] D. K. Lahiri, M. R. Farlow, N. H. Greig, and K. Sambamurti, "Current drug targets for Alzheimer's disease treatment," Drug Development Research, vol. 56, no. 3, pp. 267-281, 2002.

[4] N. C. Inestrosa, M. C. Dinamarca, and A. Alvarez, "Amyloidcholinesterase interactions implications for Alzheimer's disease," FEBS Journal, vol. 275, no. 4, pp. 625-632, 2008.

[5] H. W. Querfurth and F. M. Laferla, "Alzheimer's disease," The New England Journal of Medicine, vol. 362, pp. 329-344, 2010.

[6] World Health Organization, Neurological Disorders Public Health Challenges, World Health Organization, Geneva, Switzerland, 2006.

[7] C. Geula and M.-M. Mesulam, "Cholinesterases and the pathology of Alzheimer disease," Alzheimer Disease and Associated Disorders, vol. 9, supplement 2, pp. 23-28, 1995.

[8] M. Racchi, M. Mazzucchelli, E. Porrello, C. Lanni, and S. Govoni, "Acetylcholinesterase inhibitors: novel activities of old molecules," Pharmacological Research, vol. 50, no. 4, pp. 441451, 2004.

[9] B. Clarke, E. Demont, C. Dingwall et al., "BACE-1 inhibitors part 1: identification of novel hydroxy ethylamines (HEAs)," Bioorganic and Medicinal Chemistry Letters, vol. 18, no. 3, pp. 1011-1016, 2008.

[10] C. Gueto-Tettay, J. C. Drosos, and R. Vivas-Reyes, "Quantum mechanics study of the hydroxyethylamines-BACE-1 active site interaction energies," Journal of Computer-Aided Molecular Design, vol. 25, no. 6, pp. 583-597, 2011.

[11] P. Beswick, N. Charrier, B. Clarke et al., "BACE-1 inhibitors part 3: identification of hydroxy ethylamines (HEAs) with nanomolar potency in cells," Bioorganic and Medicinal Chemistry Letters, vol. 18, no. 3, pp. 1022-1026, 2008.

[12] N. Charrier, B. Clarke, L. Cutler et al., "Second generation of BACE-1 inhibitors. part 1: the need for improved pharmacokinetics," Bioorganic and Medicinal Chemistry Letters, vol. 19, no. 13, pp. 3664-3668, 2009.

[13] I. Bolea, J. Juárez-Jiménez, C. de los Rios et al., "Synthesis, biological evaluation, and molecular modeling of donepezil and $N$-[(5-(Benzyloxy)-1-methyl-1H-indol-2-yl)methyl]- $N$-methylprop-2-yn-1-amine hybrids as new multipotent cholinesterase/monoamine oxidase inhibitors for the treatment of Alzheimer's disease," Journal of Medicinal Chemistry, vol. 54, no. 24, pp. 8251-8270, 2011.

[14] M. L. Bolognesi, R. Banzi, M. Bartolini et al., "Novel class of quinone-bearing polyamines as multi-target-directed ligands to combat Alzheimer's disease," Journal of Medicinal Chemistry, vol. 50, no. 20, pp. 4882-4897, 2007.

[15] M. L. Bolognesi, A. Cavalli, L. Valgimigli et al., "Multi-targetdirected drug design strategy: from a dual binding site acetylcholinesterase inhibitor to a trifunctional compound against Alzheimer's disease," Journal of Medicinal Chemistry, vol. 50, no. 26, pp. 6446-6449, 2007.

[16] M. Rosini, E. Simoni, M. Bartolini et al., "Inhibition of acetylcholinesterase, $\beta$-amyloid aggregation, and NMDA receptors in Alzheimer's disease: a promising direction for the multi-targetdirected ligands gold rush," Journal of Medicinal Chemistry, vol. 51, no. 15, pp. 4381-4384, 2008.

[17] R. Carbó-Dorca, M. Arnau, and L. Leyda, "How similar is a molecule to another? An electron density measure of similarity 
between two molecular structures," International Journal of Quantum Chemistry, vol. 17, no. 6, pp. 1185-1189, 1980.

[18] R. Carbó-Dorca and L. D. Mercado, "Commentaries on quantum similarity (1): density gradient quantum similarity," Journal of Computational Chemistry, vol. 31, no. 11, pp. 2195-2212, 2010.

[19] X. Gironés and R. Carbó-Dorca, "Modelling toxicity using molecular quantum similarity measures," QSAR \& Combinatorial Science, vol. 25, no. 7, pp. 579-589, 2006.

[20] R. Carbó-Dorca, E. Besalú, and L. D. Mercado, "Communications on quantum similarity, part 3: a geometric-quantum similarity molecular superposition algorithm," Journal of Medicinal Chemistry, vol. 32, no. 4, pp. 582-599, 2011.

[21] R. Carbó-Dorca and E. Besalú, "EMP as a similarity measure: a geometric point of view," Journal of Mathematical Chemistry, vol. 51, no. 1, pp. 382-389, 2013.

[22] R. Carbó-Dorca and X. Gironés, "Foundation of quantum similarity measures and their relationship to QSPR: density function structure, approximations, and application examples," International Journal of Quantum Chemistry, vol. 101, no. 1, pp. 8-20, 2005.

[23] R. Carbó-Dorca and E. Besalú, "Communications on quantum similarity (2): a geometric discussion on holographic electron density theorem and confined quantum similarity measures," Journal of Computational Chemistry, vol. 31, no. 13, pp. 24522462, 2010.

[24] L. Amat and R. Carbó-Dorca, "Use of promolecular ASA density functions as a general algorithm to obtain starting $\mathrm{MO}$ in SCF calculations," International Journal of Quantum Chemistry, vol. 87, no. 2, pp. 59-67, 2002.

[25] P. G. Mezey, Shape in Chemistry: An Introduction to Molecular Shape and Topology, Wiley-VCH, New York, NY, USA, 1993.

[26] R. G. Parr and W. Yang, Density Functional Theory of Atoms and Molecules, Oxford University Press, New York, NY, USA, 1989.

[27] P. W. Ayers, J. S. M. Anderson, and L. J. Bartolotti, "Perturbative perspectives on the chemical reaction prediction problem," International Journal of Quantum Chemistry, vol. 101, no. 5, pp. 520-534, 2005.

[28] J. L. Gazquez, "Perspectives on the density functional theory of chemical reactivity," Journal of the Mexican Chemical Society, vol. 52, pp. 3-10, 2008.

[29] P. Geerlings, F. De Proft, and W. Langenaeker, "Conceptual density functional theory," Chemical Reviews, vol. 103, no. 5, pp. 1793-1874, 2003.

[30] W. Koch and M. C. Holthausen, A Chemist's Guide to Density Functional Theory, Wiley-VCH, Weinheim, Germany, 2nd edition, 2000.

[31] R. Vivas-Reyes, F. De Proft, P. Geerlings et al., "A DFT and $\mathrm{HF}$ quantum chemical study of the tin nanocluster $\left[(\mathrm{RSn})_{12} \mathrm{O}_{14}(\mathrm{OH})_{6}\right]^{2+}$ and its interactions with anions and neutral nucleophiles: confrontation with experimental data," New Journal of Chemistry, vol. 9, pp. 1108-1117, 2002.

[32] R. Mejia-Urueta, F. Nuñez-Zarur, and R. Vivas-Reyes, "Density functional study on electronic structures and reactivity in carbazol-oxadiazole dyads used in organic light emitting diodes," International Journal of Quantum Chemistry, vol. 112, no. 16, pp. 2808-2815, 2012.

[33] F. De Proft, R. Vivas-Reyes, M. Biesemans, R. Willem, J. M. L. Martin, and P. Geerlings, "Density functional study of the complexation reaction of $\mathrm{Sn}\left(\mathrm{CH}_{3}\right)_{3} \mathrm{X}(\mathrm{X}=\mathrm{F}, \mathrm{Cl}, \mathrm{Br}$ and I) with halide anions," European Journal of Inorganic Chemistry, vol. 2003, no. 20, pp. 3803-3810, 2003.
[34] R. Vivas-Reyes, F. De Proft, M. Biesemans, R. Willem, and P. Geerlings, "A DFT study of Tin- and crown-ether-based host molecules capable of binding anions and cations simultaneously," European Journal of Inorganic Chemistry, vol. 2003, no. 7, pp. 1315-1324, 2003.

[35] A. Morales-Bayuelo, J. Torres, and R. Vivas-Reyes, "Quantum molecular similarity analysis and quantitative definition of catecholamines with respect to biogenic monoamines associated: scale alpha and beta of quantitative convergence," International Journal of Quantum Chemistry, vol. 112, no. 14, pp. 2637-2642, 2012.

[36] A. Morales-Bayuelo, J. Torres, and R. Vivas-Reyes, "Hückel treatment of pyrrole and pentalene as a function of cyclopentadienyle using local quantum similarity index (LQSI) and the topo-geometrical superposition approach (TGSA)," Journal of Theoretical and Computational Chemistry, vol. 11, article 223, 2012.

[37] G. S. Hammond, "A correlation of reaction rates," Journal of the American Chemical Society, vol. 77, no. 2, pp. 334-338.

[38] M. Duran and J. Beltran, "Potential energy hypersurfaces," in Reports in Molecular Theory, vol. 1, p. 57, 1990.

[39] G. A. Arteca and P. G. Mezey, "Validity of the Hammond postulate and constraints on general one-dimensional reaction barriers," Journal of Computational Chemistry, vol. 9, no. 7, pp. 728-744, 1988.

[40] J. Cioslowski, "Quantifying the Hammond postulate: intramolecular proton transfer in substituted hydrogen catecholate anions," Journal of the American Chemical Society, vol. 113, no. 18, pp. 6756-6760, 1991.

[41] A. Morales-Bayuelo, H. Ayazo, and R. Vivas-Reyes, "Threedimensional quantitative structure-activity relationship CoMSIA/CoMFA and LeapFrog studies on novel series of bicyclo [4.1.0] heptanes derivatives as melanin-concentrating hormone receptor R1 antagonists," European Journal of Medicinal Chemistry, vol. 45, no. 10, pp. 4509-4522, 2010.

[42] M. Ahumedo, A. Díaz, and R. Vivas-Reyes, "Theoretical and structural analysis of the active site of the transcriptional regulators LasR and TraR, using molecular docking methodology for identifying potential analogues of acyl homoserine lactones (AHLs) with anti-quorum sensing activity," European Journal of Medicinal Chemistry, vol. 45, no. 2, pp. 608-615, 2010.

[43] X. Gironés and R. Carbó-Dorca, “TGSA-Flex: extending the capabilities of the Topo-Geometrical superposition algorithm to handle flexible molecules," Journal of Computational Chemistry, vol. 25, no. 2, pp. 153-159, 2003.

[44] L. Chen, "Substructure and maximal common substructure searching," in Computational Medicinal Chemistry for Drug Discovery, P. Bultinck, H. De Winter, W. Langenaeker, and J. P. Tollenaere, Eds., p. 483, Marcel Dekker, New York, NY, USA, 2003.

[45] J. P. Perdew, J. A. Chevary, S. H. Vosko et al., "Atoms, molecules, solids, and surfaces: applications of the generalized gradient approximation for exchange and correlation," Physical Review B, vol. 46, no. 11, pp. 6671-6687, 1992, Erratum in Physical Review $B$, vol. 48, p. 4978, 1993.

[46] J. P. Perdew, K. Burke, and Y. Wang, "Generalized gradient approximation for the exchange-correlation hole of a manyelectron system," Physical Review B, vol. 54, pp. 16533-16539, 1996, Erratum in Physical Review B, vol. 57, p. 14999, 1998.

[47] J. P. Perdew, K. Burke, and M. Ernzerhof, "Generalized gradient approximation made simple," Physical Review Letters, vol. 77, no. 18 , pp. 3865-3868, 1996. 
[48] A. D. Becke, "Density-functional exchange-energy approximation with correct asymptotic behavior," Physical Review A, vol. 38, no. 6, pp. 3098-3100, 1988.

[49] A. D. Becke, "A new inhomogeneity parameter in densityfunctional theory," Journal of Chemical Physics, vol. 109, no. 6, article 2092, 1998.

[50] W. J. Hehre, L. Radom, P. V. R. Schleyer, and J. A. Pople, Ab Initio Molecular Orbital Theory, John Wiley \& Sons, New York, NY, USA, 1986.

[51] M. J. Frisch, G. W. Trucks, H. B. Schlegel et al., "GAUSSIAN 09, Revision A. 02," Gaussian, Wallingford, UK, 2009.

[52] P. Bultinck, X. Girones, and R. Carbó-Dorca, "Molecular quantum similarity: theory and applications," Reviews in Computational Chemistry, vol. 21, 2005.

[53] P. Bultinck and R. Carbó-Dorca, "A mathematical discussion on density and shape functions, vector semispaces and related questions," Journal of Mathematical Chemistry, vol. 36, no. 2, pp. 191-200, 2004.

[54] E. W. Weisstein, Encyclopedia of Mathematics, Chapman \& Hall, London, UK, 2003.

[55] F. L. Hirshfeld, "Bonded-atom fragments for describing molecular charge densities," Theoretica Chimica Acta, vol. 44, no. 2, pp. 129-138, 1977.

[56] R. F. Nalewajski and R. G. Parr, "Information theory, atoms in molecules, and molecular similarity," Proceedings of the National Academy of Sciences of the United States of America, vol. 97, no. 16, pp. 8879-8882, 2000.

[57] F. De Proft, R. Vivas-Reyes, A. Peeters, C. Van Alsenoy, and P. Geerlings, "Hirshfeld partitioning of the electron density: atomic dipoles and their relation with functional group properties," Journal of Computational Chemistry, vol. 24, no. 4, pp. 463-469, 2003.

[58] P. G. Mezey, "The holographic electron density theorem and quantum similarity measures," Molecular Physics, vol. 96, no. 2, pp. 169-178, 1999.

[59] P. Constans and R. Carbo, "Atomic shell approximation: electron density fitting algorithm restricting coefficients to positive values," Journal of Chemical Information and Computer Sciences, vol. 35, no. 6, pp. 1046-1053, 1995.

[60] R. Carbó-Dorca, "Quantum similarity, volume functions and generalized Carbó indices," Journal of Mathematical Chemistry, vol. 49, no. 9, pp. 2109-2115, 2011.

[61] R. G. Parr and R. G. Pearson, "Absolute hardness: companion parameter to absolute electronegativity," Journal of the American Chemical Society, vol. 105, no. 26, pp. 7512-7516, 1983.

[62] R. G. Pearson, Chemical Hardness, Wiley-VCH, Weinheim, Germany, 1997.

[63] P. W. Ayers, "The physical basis of the hard/soft acid/base principle," Faraday Discuss, vol. 135, pp. 161-190, 2007.

[64] W. T. Yang and R. G. Parr, "Hardness, softness, and the fukui function in the electronic theory of metals and catalysis," Proceedings of the National Academy of Sciences, vol. 82, pp. 6723-6726, 1985.

[65] A. K. Chandra and M. T. Nguyen, "Fukui function and local softness as reactivity descriptors," in Chemical Reactivity Theory: A Density-Functional View, P. K. Chattaraj, Ed., p. 163, Taylor and Francis, New York, NY, USA, 2008.

[66] R. G. Parr and W. T. Yang, "Density functional approach to the frontier-electron theory of chemical reactivity," Journal of the American Chemical Society, vol. 106, no. 4, pp. 4049-4050, 1984.
[67] W. T. Yang, R. G. Parr, and R. Pucci, "Electron density, Kohn-Sham frontier orbitals, and Fukui functions," Journal of Chemical Physics, vol. 81, no. 6, article 2862, 1984.

[68] P. W. Ayers, W. T. Yang, and L. J. Bartolotti, "The Fukui function," in Chemical Reactivity Theory: A Density Functional View, P. K. Chattaraj, Ed., p. 255, CRC Press, Boca Raton, Fla, USA, 2009.

[69] P. W. Ayers and M. Levy, "Perspective on 'density functional approach to the frontier-electron theory of chemical reactivity", Theoretical Chemistry Accounts, vol. 103, no. 3-4, pp. 353-360, 2000.

[70] K. Fukui, "Role of frontier orbitals in chemical reactions," Science, vol. 217, pp. 747-754, 1982.

[71] K. Fukui, T. Yonezawa, and H. Shingu, "A molecular orbital theory of reactivity in aromatic hydrocarbons," The Journal of Chemical Physics, vol. 20, no. 4, pp. 722-725, 1952.

[72] W. Yang and W. J. Mortier, "The use of global and local molecular parameters for the analysis of the gas-phase basicity of amines," Journal of the American Chemical Society, vol. 108, no. 19 , pp. 5708-5711, 1986.

[73] A. D. McLean and G. S. Chandler, "Contracted Gaussian basis sets for molecular calculations. I. Second row atoms, $Z=11-18$," The Journal of Chemical Physics, vol. 72, no. 10, pp. 5639-5648, 1980.

[74] J. D. Barrow, The World within the World, chapter 3, Clarendon Press, Oxford, UK, 1988.

[75] G. Ucar, N. Gokhan, A. Yesilada, and A. A. Bilgin, "1$N$ thiocarbamoyl-3-phenyl-5-thienyl-2-pyrazolines: a novel cholinesterase and selective monoamine oxidase B inhibitors for the treatment of Parkinson's and Alzheimer's diseases," Neuroscience Letters, vol. 382, no. 3, pp. 327-331, 2005.

[76] J. Moizeszowicz, Psicofarmacología Psicodinámica IV, Editorial Paidós, Buenos Aires, Argentina, 2000.

[77] W. Maruyama, M. Weinstock, M. B. H. Youdim, M. Nagai, and M. Naoi, "Anti-apoptotic action of anti-Alzheimer drug, TV3326 [(N-propargyl)-(3R)-aminoindan-5-yl]-ethyl methyl carbamate, a novel cholinesterase-monoamine oxidase inhibitor," Neuroscience Letters, vol. 341, no. 3, pp. 233-236, 2003.

[78] T. Wilens and J. Biederman, “The stimulants," Psychiatric Clinics of North America, vol. 15, no. 1, pp. 191-222, 1992.

[79] J. Sterling, Y. Herzig, T. Goren et al., "Novel dual inhibitors of AChE and MAO derived from hydroxy aminoindan and phenethylamine as potential treatment for Alzheimer's disease," Journal of Medicinal Chemistry, vol. 45, no. 24, pp. 5260-5279, 2002.

[80] J. Sterling, A. Veinberg, D. Lerner et al., “(R)(+)-N-propargyl1-aminoindan (rasagiline) and derivatives: highly selective and potent inhibitors of monoamine oxidase B," Journal of Neural Transmission. Supplementa, vol. 52, pp. 301-305, 1998.

[81] E. Groner, Y. Ashani, D. Schorer-Apelbaum, J. Sterling, Y. Herzig, and M. Weinstock, "The kinetics of inhibition of human acetylcholinesterase and butyrylcholinesterase by two series of novel carbamates," Molecular Pharmacology, vol. 71, pp. 16101617, 2007.

[82] A. Ordentlich, D. Barak, C. Kronman et al., "The architecture of human acetylcholinesterase active center probed by interactions with selected organophosphate inhibitors," The Journal of Biological Chemistry, vol. 271, pp. 11953-11962, 1996.

[83] M. A. Mena, E. G. Aguado, and J. G. De Yebenes, "Monoamine metabolites in human cerebrospinal fluid. HPLC/ED method," Acta Neurologica Scandinavica, vol. 69, no. 4, pp. 218-225, 1984. 

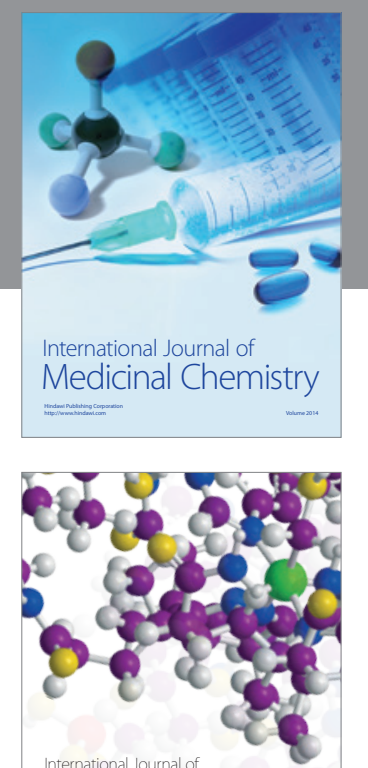

\section{Carbohydrate} Chemistry

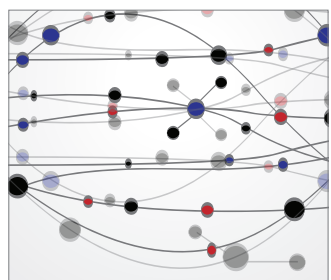

The Scientific World Journal
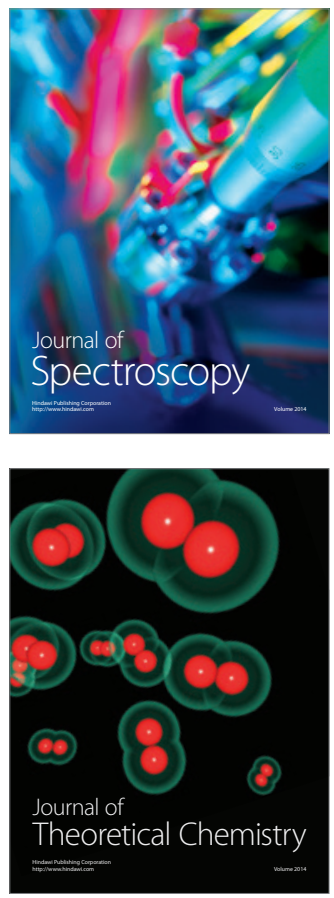
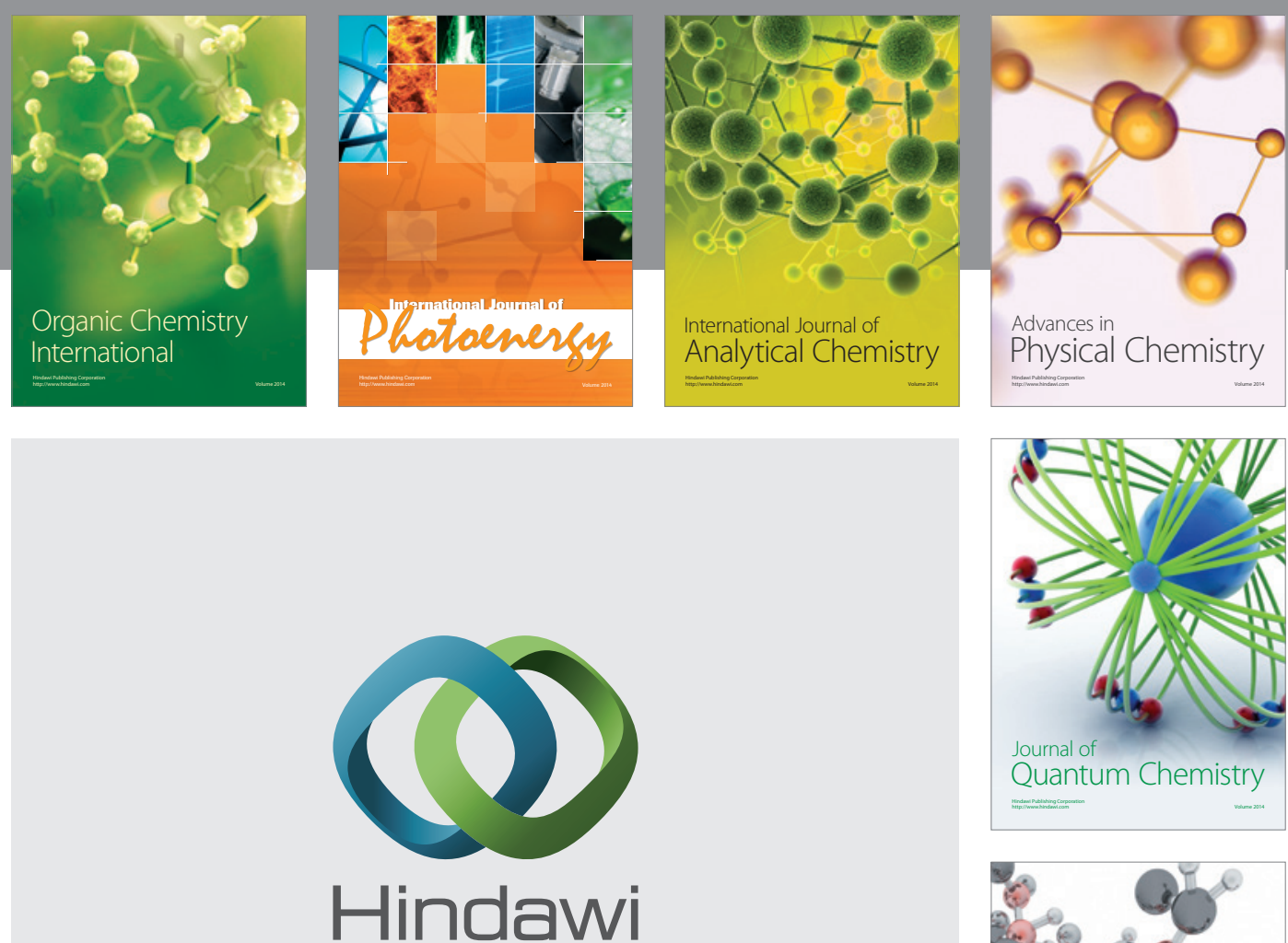

Submit your manuscripts at

http://www.hindawi.com

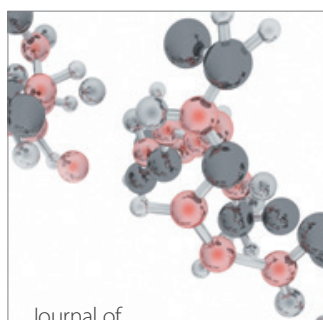

Analytical Methods

in Chemistry

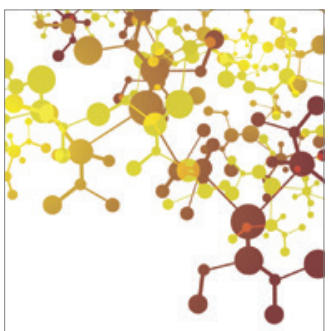

Journal of

Applied Chemistry

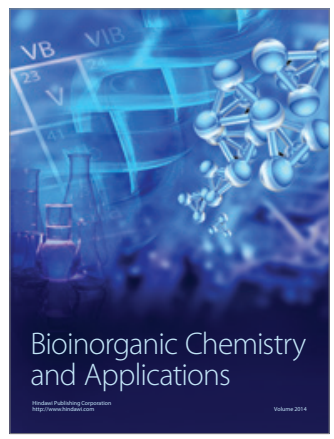

Inorganic Chemistry
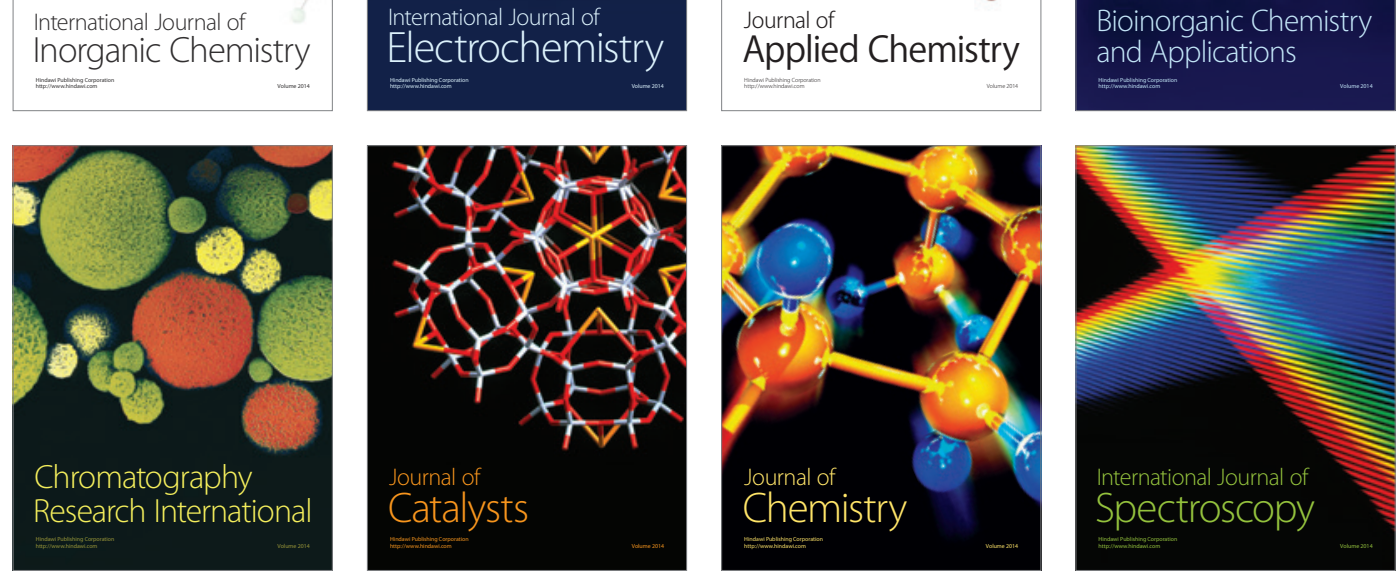